\title{
Overview of South African Dendromonocotyle (Monogenea: Monocotylidae), with descriptions of 2 new species from stingrays (Dasyatidae) kept in public aquaria
}

\author{
DAVID VAUGHAN ${ }^{1,2}$, LESLIE CHISHOLM $^{3} \&$ KEVIN CHRISTISON ${ }^{1,4}$ \\ ${ }^{1}$ Department of Biodiversity and Conservation Biology, University of the Western Cape, Private Bag X 17, Bellville, 7535, South \\ Africa.E-mail:dvaughan@aquarium.co.za; kchris@deat.gov.za \\ ${ }^{2}$ Two Oceans Aquarium, P.O. Box 50603, Victoria \& Alfred Waterfront, Cape Town, 8000, South Africa \\ ${ }^{3}$ Marine Parasitology Laboratory, School of Earth and Environmental Sciences, (DX650 418), The University of Adelaide, North Ter- \\ race, Adelaide, South Australia 5005, Australia.E-mail: chisholm.leslie@saugov.sa.gov.au \\ ${ }^{4}$ Department of Environmental Affairs and Tourism, Marine and Coastal Management, Private Bag X2, Roggebaai, 8012, South Africa
}

\begin{abstract}
Two new species, Dendromonocotyle citrosa n. sp. off Dasyatis chrysonota (Smith) from Two Oceans Aquarium in Cape Town and off Himantura gerrardi (Gray) from uShaka Sea World in Durban and D. ukuthena n. sp. off H. gerrardi and H. uarnak (Forsskål) also from uShaka Sea World in Durban, are described. These can be distinguished from previously described Dendromonocotyle species by the morphology of the distal portion of the male copulatory organ and the proximal part of the vagina. Vaginal morphology is proposed as an important diagnostic character for species in the genus. Dendromonocotyle colorni Chisholm, Whittington \& Kearn, 2001 which was originally recorded from H. uarnak in Israel, was discovered on $H$. uarnak sympatrically with $D$. ukuthena n. sp. and with $D$. citrosa n. sp. and $D$. ukuthena n. sp. off $H$. gerrardi. Minor differences in the number of papillary sclerites, the presence of septal tripartite sclerites and in the number of eyespots were seen between $D$. colorni collected from H. uarnak and H. gerrardi from uShaka Sea World and those originally described off $H$. uarnak from Israel. We demonstrate that the position of the marginal hooklets can be used to determine the orientation of the haptor of all representative Dendromonocotyle species with or without hamuli. We conclude that Dendromonocotyle species may not be as host-specific as previously believed and that minor differences in morphology are host or geographically induced.
\end{abstract}

Key words: Dendromonocotyle, Monogenea, Monocotylidae, Himantura, Dasyatis, public aquaria, stingrays

\section{Introduction}

Members of Dendromonocotyle Hargis, 1955 are usually found exclusively on the dorsal skin surface of stingrays and the genus currently comprises 13 species (Chisholm et al. 2005). Intensities of monogeneans on wild sharks and stingrays are generally lower than those kept in captivity (Chisholm et al. 2004). Because they have a direct life-cycle, monogeneans can be particularly important disease-causing organisms on captive hosts due to their ability to increase rapidly in numbers (Chisholm et al. 2004). Infection potential is further enhanced since the density of hosts in aquarium exhibits is usually high. Lesions caused from large numbers of feeding monogeneans can lead to opportunistic secondary infections of the host by bacterial pathogens, or can be directly responsible for host mortality (Bullard et al. 2001). Indeed, high intensities of Dendromonocotyle species infesting stingrays in public aquaria have been shown to be problematic (see Chisholm et al. 2004). 